\title{
Molecular Pathways Controlling Development of Thalamus and Hypothalamus: From Neural Specification to Circuit Formation
}

\author{
Seth Blackshaw, ${ }^{1,2,3,4,5}$ Steffen Scholpp, ${ }^{6}$ Marysia Placzek, ${ }^{7}$ Holly Ingraham, ${ }^{8}$ Richard Simerly, ${ }^{9}$ \\ and Tomomi Shimogori ${ }^{10}$ \\ Departments of ${ }^{1}$ Neuroscience, ${ }^{2}$ Neurology, and ${ }^{3}$ Ophthalmology, and ${ }^{4}$ Institute for Cell Engineering and ${ }^{5}$ Center for High Throughput Biology, Johns \\ Hopkins University School of Medicine, Baltimore, Maryland 21287, ${ }^{6}$ Department of Toxicology, Karlsruhe Institute of Technology, Institute for Toxicology \\ and Genetics, 76344 Karlsruhe, Germany, ${ }^{7}$ Medical Research Council Centre for Developmental and Biomedical Genetics and Department of Biomedical \\ Science, University of Sheffield, Sheffield S10 2TN, United Kingdom, ${ }^{8}$ Department of Physiology, University of California, San Francisco, San Francisco, \\ California 94143, ${ }^{9}$ The Saban Research Institute, Childrens Hospital Los Angeles, Keck School of Medicine, University of Southern California, Los Angeles, \\ California 90027, and ${ }^{10}$ RIKEN Brain Science Institute, Wako, Saitama 351-0198, Japan
}

The embryonic diencephalon gives rise to the vertebrate thalamus and hypothalamus, which play essential roles in sensory information processing and control of physiological homeostasis and behavior, respectively. In this review, we present new steps toward characterizing the molecular pathways that control development of these structures, based on findings in a variety of model organisms. We highlight advances in understanding how early regional patterning is orchestrated through the action of secreted signaling molecules such as Sonic hedgehog and fibroblast growth factors. We address the role of individual transcription factors in control of the regional identity and neural differentiation within the developing diencephalon, emphasizing the contribution of recent large-scale gene expression studies in providing an extensive catalog of candidate regulators of hypothalamic neural cell fate specification. Finally, we evaluate the molecular mechanisms involved in the experience-dependent development of both thalamo-cortical and hypothalamic neural circuitry.

\section{Introduction}

The developing vertebrate forebrain consists of two major parts: the telencephalon-which gives rise to cerebral cortex, striatum, amygdala, and associated structures - and the diencephalon. The diencephalon gives rise to two essential brain regions, the thalamus and hypothalamus (Fig. $1 A$ ). Though both structures are derived from a common region of the anterior neural tube, they serve very different physiological functions. The thalamus acts as a central integrator of sensory information, receiving afferents from receptors of all sensory modalities save olfaction, and serves as the sole path by which integrated sensory information reaches the cerebral cortex and gives rise to conscious perception. The hypothalamus, on the other hand, serves a more diverse range of functions. It is a central regulator of critical homeostatic physiological processes, including temperature regulation, food intake,

Received Aug. 26, 2010; revised Aug. 31, 2010; accepted Sept. 1, 2010.

This work was supported by a Basil O'Connor Starter Scholar Award from the March of Dimes (S.B.), an award from the Klingenstein Fund (S.B.), and the RIKEN Brain Science Institute and Human Frontier Science Project (T.S.). S.B. is a W. M. Keck Distinguished Young Scholar in Medical Research.

We thank J. Bedont and W. Yap for comments on the manuscript.

Correspondence should be addressed to either of the following: Seth Blackshaw, Departments of Neuroscience, Neurology, and Ophthalmology, and Institute for Cell Engineering and Center for High-throughput Biology, Johns Hopkins University School of Medicine, Baltimore, MD 21287, E-mail: sblack@jhmi.edu; or Tomomi Shimogori, RIKEN Brain Science Institute, 2-1 Hirosawa Wako, Saitama 351-0198, Japan, E-mail: tshimogori@brain.riken.jp.

DOI:10.1523/JNEUROSCI.4499-10.2010

Copyright $\odot 2010$ the authors $\quad 0270-6474 / 10 / 3014925-06 \$ 15.00 / 0$ and circadian rhythms, which it accomplishes in part by acting as the central regulator of the neuroendocrine system. It is also critical in the processing of many different emotional and social behaviors, including mating, fighting, and parental care. Finally, it plays a critical though still little-studied role in memory formation.

Both thalamus and hypothalamus are comprised of a highly diverse collection of cell groups and neuronal subtypes. This anatomical complexity, and the lack of well defined molecular markers that delineate particular cell types, has traditionally held back studies of these structures' development, particularly compared with other CNS regions such as the spinal cord, retina and cerebral cortex. With this in mind, these presentations aim to stimulate interaction between experts in this field and the broader neuroscience community, and encourage other researchers to enter this young and exciting field.

Patterning of developing thalamus and hypothalamus by secreted differentiation factors

Studies of early diencephalic patterning over the last 2 decades characterized a relatively small number of gene sets expressed in specific compartments of the developing diencephalon (Figdor and Stern, 1993; Puelles and Rubenstein, 1993). These gene sets were used to generate the prosomeric model, a representation of the hypothesized morphological boundaries of the developing diencephalon (Puelles and Rubenstein, 2003). The prosomeric 
model identifies three major compartments within the developing diencephalon (p1, p2 and p3, moving posterior to anterior) along the longitudinal axis. A hallmark of this model is the clear boundary that separates $\mathrm{p} 2$ and $\mathrm{p} 3$, termed the zona limitans intrathalamica (Zli), which includes the mid-diencephalic organizer (MDO) (Scholpp et al., 2009). This structure releases several secreted factors essential for patterning the developing thalamus, including Sonic hedgehog (Shh), and members of the Wnt and fibroblast growth factor (Fgf) family (Fig. 1A) (Bulfone et al., 1993).

Selective expression of hedgehog family genes in the MDO of vertebrate model organisms is observed in lamprey (Osorio et al., 2005), zebrafish (Macdonald et al., 1995), frog (Ruiz i Altaba, 1998), chick (Kitamura et al., 1997), and mouse (Kitamura et al., 1997). Experimental abrogation of Shh signaling in zebrafish, chick, and mouse results in loss of genetic fate determinants and cell identity in prethalamus and thalamus (Kiecker and Lumsden, 2004; Scholpp et al., 2006; Vue et al., 2009). Furthermore, ectopic activation of the Shh pathway by misexpression of a constitutively active Shh effector mutant (SmoM2) induces the expression of thalamic markers such as Gbx2, Ngn2, Olig2, and Olig3 in the mouse pretectum (Vue et al., 2009). These studies showed that Shh induces the expression of two proneural genes within the developing sensory thalamus: a posterior-to-anterior wave of Ngn1 expression in the major caudal thalamus and a narrow stripe of Ascl1 expression in rostral thalamic cells immediately adjacent to the MDO (Fig. $1 B, C$ ). Blockade of Shh signaling leads to both a substantial decrease in the size of the Ngn1-positive caudal thalamus and the absence of the Ascl1positive rostral thalamus (Vue et al., 2009).

How does Shh signaling selectively induce expression of these neurogenic basic helix-loop-helix genes in the two major divisions of the developing sensory thalamus? Studies in zebrafish have shown that the effect of Shh is controlled by the selector gene Her6, a homolog of the mouse Hes1 gene (Fig. 1A). Her6 is initially broadly expressed throughout thalamic neuroepithelium but is later progressively lost from the caudal thalamus. This creates a dynamic posterior-to-anterior "neurogenetic gradient" of $\mathrm{Ngn} 1$ expression in the thalamus. Subsequently, the remaining anterior Her6 induces Ascl1 expression in a stripe of rostral thalamic cells. This zonation of proneural gene expression is followed by posterior-to-anterior differentiation of glutamatergic relay neurons from the Ngn1-positive precursors in the caudal relay thalamus and of GABAergic inhibitory neurons from the Ascl1-expressing precursors in the reticular nucleus of the rostral thalamus. Therefore, the dynamic expression of Her6 defines neurotransmitter fates within the thalamus (Scholpp et al., 2009), and release of Shh determines the time point at which proneural gene expression, and subsequent neuronal differentiation, initiates within the developing thalamus.

In parallel to the Shh pathway, Fgf8 expression anterior to the MDO controls the anterior and posterior identity of thalamic
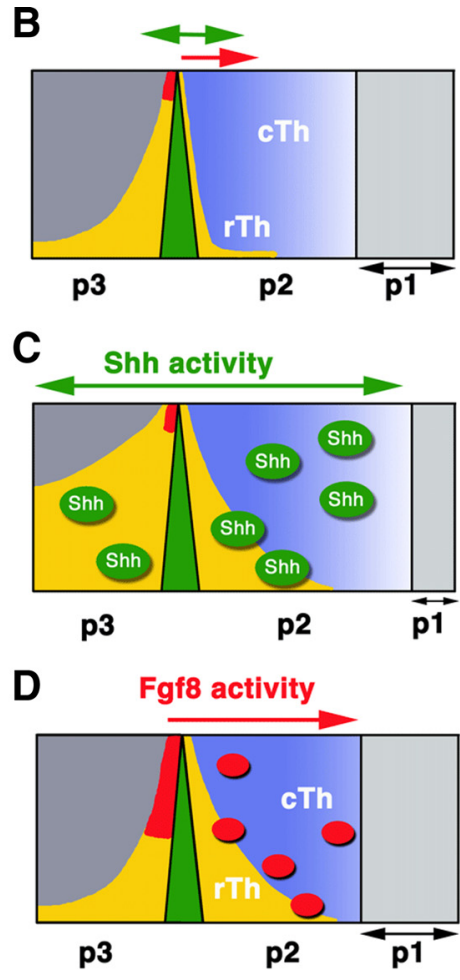

Figure 1. $\quad \boldsymbol{A}$, Sagittal schematic of mouse embryonic brain at E10.5. The diencephalic neuraxis is divided in p1, p2, p3, and al diencephalon and hypothalamus are indicated in red and Hes 1 (Her6) and Ascl1 (acc1) are indicated in yellow. B Schema al diencephalon is detectable only in 3 (red). The 2 region an be further subdivided in is detectable only posterior to this region. C, Overexpression of Shh expands the Hes1 and Ascl1 in both p2 and p3. D, Overexpression of Fgf8 expands the Hes1 and Ascl1 only in p2.

nuclei (Kataoka and Shimogori, 2008). Using in utero electroporation to overexpress or inhibit endogenous Fgf8 in the mouse MDO, differentiation of two distinct progenitor populations in p2, marked respectively by Ngn2 and Ascl1 expression, were found to be regulated by Fgf signaling in a complementary manner (Fig. 1D). Furthermore, increased FGF activity shifts thalamic sensory nuclei posteriorly in postnatal mouse thalamus, which suggests that Fgf8 originating anterior to the MDO controls pattern formation in the region posterior to the MDO. However, the precise molecular mechanism by which Fgf8 controls diencephalic pattern and how Shh and FGF8 interact to correctly pattern the nascent thalamus has not yet been established.

Classical signaling factors are also expressed in spatially restricted fashions in the developing hypothalamus and play an essential role in its development and patterning (Fig. $1 \mathrm{~A}$ ). A Wnt activity gradient, from posterior to anterior, appears to govern the early acquisition of ventral hypothalamic identity: zebrafish embryos mutant for Axin1, an intracellular negative regulator of Wnt pathway activity, show a reduction of prospective ventral hypothalamic tissue and concomitant expansion of floor plate. Similarly, a reduction in Wnt signaling in the chick promotes ventral hypothalamic fate at the expense of floor plate. Wnt signaling also governs regionalization of the nascent hypothalamus, directing the emergence of posterior (mamillary) hypothalamic fates (Kapsimali et al., 2004; Manning et al., 2006). Although most Wnt ligands and components are restricted to the posterior 
A

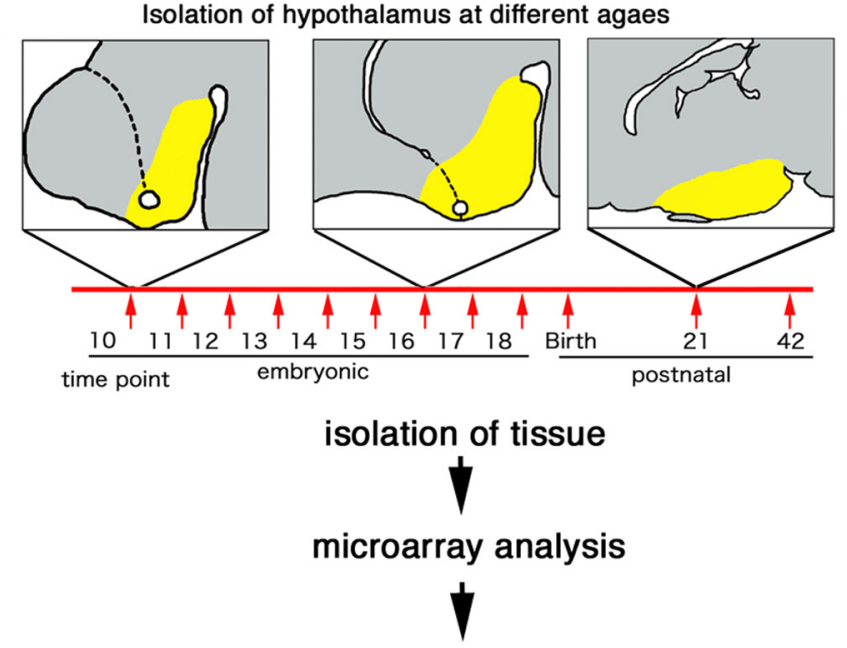

ISH at E11.5, E12.5 E14.5 and E16.5
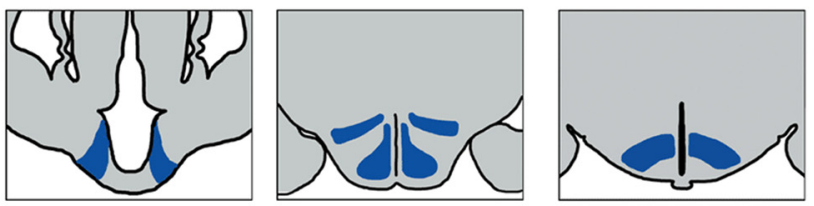

C Isolation of $\mathrm{VMH}$ at $\mathrm{PO}$

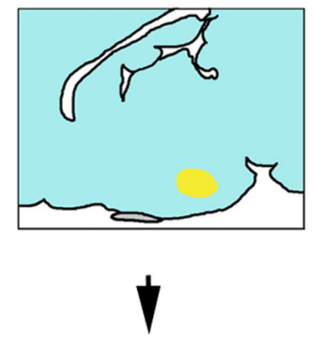

microarray analysis

$\mathrm{ISH}$ at $\mathrm{PO}$

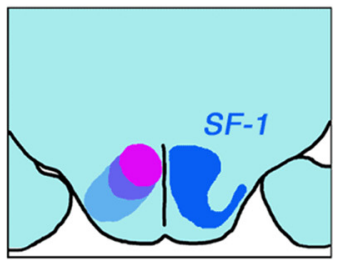

Vgll2, Sox14, Satb2

Sox14, Satb2

Satb2
B

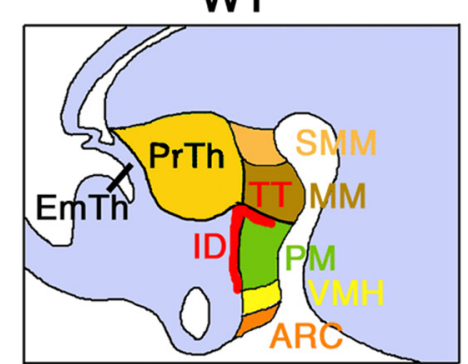

Nkx2.1 ${ }^{\text {cre }} \times S h h^{\text {loxP/loxP }}$

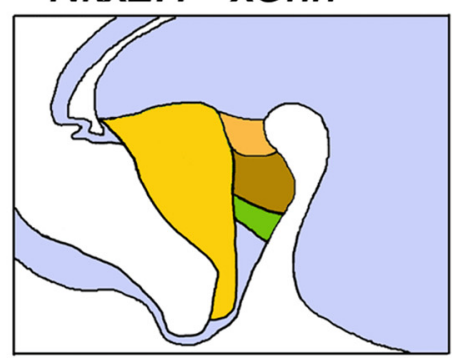

Figure 2. $\quad \boldsymbol{A}$, Combined use of microarray-based expression profiling and large-scale in situ hybridization for the construction of the genomic atlas of mouse hypothalamic development. $\boldsymbol{B}$, Developmental phenotype of deletion of Shh from hypothalamic basal plate determined using regional markers identified in $\boldsymbol{A}$. PrTh, prethalamus (yellow, anterior; and green, posterior), EmTh, eminentia thalami; SMM, supramamillary nucleus; MM, mamillary nucleus; PM, premamillary nucleus; TT, tuberomamillary terminal. $\boldsymbol{C}$, Schematic for expression profiling of developing VMH in neonatal mice. Subdomains of VMH expressing different combinations of transcripts are indicated.

hypothalamus throughout development, Shh and multiple different bone morphogenetic proteins (BMPs) show dynamic spatio-temporal profiles. Expression of Shh and Shh signaling components is negatively regulated by BMP signaling (Manning et al., 2006; Ohyama et al., 2008). Studies in the limb bud show that cross talk between the BMP and Shh pathways is critical for integrated growth, pattern, and differentiation (Bénazet et al., 2009), and raises the possibility that a similar cross talk between BMP and Shh signaling regulates proliferation and spatial patterning of hypothalamic neural progenitors in a concerted manner. In support of this hypothesis, Shh is critical for the proliferation and patterning of anterior hypothalamic neural progenitors (Dale et al., 1997; Manning et al., 2006; Szabó et al., 2009; Shimogori et al., 2010), whereas BMP7 signaling regulates the proliferation and spatial identity of posteroventral hypothalamic progenitors. Forced maintenance of Shh and Shh signaling components abolishes posterior identities, defined through expression of the transcription factor Emx2 (Manning et al., 2006). The BMP-mediated downregulation of Shh expression leads to expression of Fgf family members Fgf8 and Fgf10, showing strong expression in the ventral hypothalamic floor following downregulation of Shh expression. Current studies are addressing the potential role of FGF signaling from the hypothalamic floor, demonstrating that it regulates the proliferation of progenitors in the mid (tuberal)-hypothalamus (Manning et al., 2006). Intriguingly, the spatially restricted expression of FGF and Shh along the hypothalamic dorsoventral axis not only persists throughout embryogenesis but is maintained into adulthood, suggesting that their activities might control progenitor cell proliferation in the adult hypothalamus.

\section{Transcriptional control of cell identity in developing} hypothalamus: from molecular atlas construction to candidate gene analysis

Secreted differentiation factors such as those described above confer identity on neural subtypes within the developing nervous system by inducing expression of regional and cell type-specific transcription factors. However, two major obstacles to the systematic analysis of hypothalamic development have been the lack of well defined molecular markers for identifying individual cell types, along with a dearth of candidate transcription factors that might direct specification of hypothalamic neuronal subtypes. To 
overcome these obstacles, several largescale efforts have been undertaken to comprehensively profile changes in gene expression both globally in the developing hypothalamus and in individual developing hypothalamic nuclei. In one such study, aimed at globally profiling genes expressed in developing mouse hypothalamus, microarray analysis of whole hypothalamic neuroepithelium at 12 different developmental time points was conducted. This was then followed by single-color and two-color in situ hybridization of $>1000$ transcripts in developing hypothalamus that showed dynamic expression by microarray analysis (Fig. $2 A)$. From this analysis, multiple markers that stably label each major hypothalamic nucleus over the entire course of neurogenesis were identified (Shimogori et al., 2010). This detailed analysis not only identified hundreds of previously uncharacterized region and cell subtype-specific molecular markers but also revealed several previously uncharacterized domains within the developing hypothalamus. One notable novel compartment, termed the intra-hypothalamic diagonal (ID), consists of a zone of Arxpositive, Gad67-positive cells that separates the anteriodorsal and posterioventral portions of the hypothalamus This region expresses multiple Lhx family genes along its anterior-posterior axis and may give rise to the bulk of hypothalamic interneurons (Fig. $2 B$ ). Using this comprehensive set of region-specific hypothalamic markers, it became possible to directly analyze the effects of removal of Shh from the basal plate domain of the developing hypothalamus using Nkx2.1-Cre $x$ Shh ${ }^{\text {lox/lox }}$ mice (Fig. 2 B). Loss of this source of Shh resulted in a failure of anterior and tuberal hypothalamic nuclei to develop, along with cells of the ID, whereas development of mamillary hypothalamus and prethalamus were unaffected.

While very few genes are expressed exclusively in developing hypothalamus, it is possible using this genomic developmental atlas to identify many combinatorial patterns of gene expression that uniquely define various hypothalamic regions and cell types. For instance, $L h x 1$ and $L h x 8$, which regulate neuronal specification in other brain regions (Zhao et al., 1999; Mori et al., 2004; Fragkouli et al., 2005; Zhao et al., 2007), are both expressed in the anterior hypothalamic neuroepithelium at embryonic day (E) 12.5. Later, at the time when hypothalamic neurogenesis ends (E16.5), Lhxl expression is restricted to the developing SCN (suprachiasmatic nucleus) and $L h x 8$ expression is restricted to the central DMH (dorsomedial hypothalamic nucleus). By generating Six3-Cre $x \operatorname{Lh} \times 1^{10 x / l o x}$ mice, in which $L h x 1$ is selectively deleted from anterior hypothalamus, it was found that both terminal differentiation of SCN neurons and circadian behavioral rhythms are severely disrupted.

Similar efforts by other investigators have focused on analyzing development of the ventromedial hypothalamic nucleus $(\mathrm{VMH})$, since it is linked to many innate behavioral responses, including feeding, fear, thermoregulation, and sexual activity, and can be readily and uniquely identified by expression of the nuclear hormone receptor Nr5a1. The precise neuronal circuits underlying these innate responses are largely unknown, in part because the cellular and molecular features of individual VMH neuronal subtypes have yet to be defined.

To this end, using laser-capture microdissection of neonatal $\mathrm{VMH}$ combined with microarray-based expression profiling (Fig. 2C), novel molecular markers of $\mathrm{VMH}$ in the newborn mouse were identified (Kurrasch et al., 2007). Many of these neuroendocrine markers exhibit distinct regional expression patterns in the newly formed hypothalamus. Top neonatal markers include transcriptional regulators such as Vgll2, Nr5a1, Sox14, Satb2, Fezf1, Dax1, Nkx2-2, and COUP-TFII. Interestingly, the highest expressed VMH transcript, the coregulator Vgll2, is completely absent in older animals. All VMH progenitors, as marked by steroidogenic factor 1 (SF-1; Nr5al) expression, coexpress $N k x 2.1$ on E11.5. Later in development $N k x 2.1$ and SF-1 expression are non-overlapping. This is consistent with cellular studies in embryonic immortalized hypothalamic cells showing that $N r 5 a 1$ potently represses $N k \times 2.1$ expression (Tran et al., 2003).

This new molecular toolkit has made it possible to investigate how neuronal subtypes of the $\mathrm{VMH}$ are specified and how they might contribute to innate behavioral responses. Floxed alleles of genes prominently and selectively expressed in developing VMH have been obtained and can be crossed with Nr5al Cre-driver lines to selectively eliminate gene function in the VMH. These VMH mutant mice have been subsequently crossed with a Nr5al-green fluorescent protein (GFP) knock-in reporter line that marks all major VMH projections. Strikingly, some of these GFP-positive projections exist as early as E11.5, including fibers to the habenular nucleus and thalamus via the ventral supraoptic commissure. Data assessing morphology, $\mathrm{VMH}$ projections, and behavior are presented on mice harboring conditional knock-outs of several genes, including Nkx2.1, A2BP1, VGlut2, and Fbxw7. These studies lead us to propose that the $\mathrm{VMH}$ is an important brain center for regulation of anxiety-related behavior. 


\section{Experience-dependent development of neural circuitry in thalamus and hypothalamus}

The activity of thalamocortical axons (TCAs) has a critical role in shaping the receptive fields of neurons in layer IV of primary sensory cortex. Although this is most widely known and studied in the context of the ocular dominance columns of primate and carnivore visual cortex, more genetically tractable model organisms can also be used to investigate the mechanisms that regulate this process. In rodents, the arrangement of whiskers on the face is precisely mapped onto barrel-shaped receptive fields in layer IV of the somatosensory cortex. The barrel field contains spiny stellate cells, located predominantly around the thalamocortical axon-rich barrel centers (Fig. 3A). Each spiny stellate cell sends dendrites into a single barrel, and postsynaptic NMDA activity is critical for spiny stellate dendrite formation (Iwasato et al., 2000; Espinosa et al., 2009). Furthermore, serotonergic autoregulation has an important role in both regulating targeting of somatosensory TCAs and in barrel field formation, as determined by analysis of mice mutant for genes involved in serotonin biosynthesis or reuptake (Rebsam et al., 2002). Perturbations of the sensory periphery by nerve or whisker lesions during a critical period disrupt barrel structure, suggesting that early neural activity is essential for pattern formation in the barrel cortex (Lendvai et al., 2000). These results suggest that cell organization and dendrite formation of neurons in barrel cortex are dependent on TCA innervation and activity.

To reveal the molecular mechanisms that control barrel formation by thalamocortical input, gene expression profiles were obtained from the somatosensory cortex and thalamus of mice that had undergone neonatal whisker removal, and compared with profiles obtained from the contralateral unoperated hemisphere (Fig. 3A). Following this treatment, 103 transcripts are downregulated and 100 transcripts are significantly upregulated by $>1.2$-fold in the cortex of the experimental hemisphere attributable to abnormal input from the TCAs. Therefore, thalamocortical innervation of the barrel field affects gene expression patterns in developing somatosensory cortex, altering both the position of neuronal cell bodies and dendritic development. Functional analysis of these genes will provide more detailed insight into the molecular mechanisms that control the remodeling of dendritic arbors and receptive fields of cortical neurons in response to sensory input.

Dietary and endocrine signals can likewise profoundly affect the development of neural circuitry in the hypothalamus during the perinatal period, resulting in changes that can drastically affect behavioral homoeostasis throughout the later life of the individual. Links between the perinatal nutritional environment and metabolic phenotype have been known for decades; being born of either low or high birth weight confers an increased risk for obesity and diabetes. Manipulation of litter size also alters levels of the adipocyte-derived hormone leptin, which has been identified as a potent neurotrophic factor that functions during perinatal life to specify patterns of connectivity in the hypothalamus (Simerly, 2008).

During hypothalamic development, axons travel from the arcuate nucleus (ARC) to restricted targets and distribute to form synaptic contacts with neurons in the paraventricular nucleus of the hypothalamus (PVH), which contains cells known to mediate multiple aspects of energy balance (Fig. $3 B$ ). Neural projections from the ARC to functionally distinct parts of the PVH develop during a postnatal period when leptin levels are elevated, and ARC projection pathways are severely disrupted in leptindeficient (ob/ob) mice (Fig. 3B) (Bouret et al., 2004). Treatment of $o b / o b$ mice with leptin in adulthood does not restore the normal pattern of projections, but the pathways are largely rescued by neonatal leptin treatments. Thus, the postnatal leptin surge appears to be a key developmental signal that affects the architecture of hypothalamic circuits that mediate feeding during a discrete developmental critical period. Although leptin impacts both anorexigenic proopiomelanocortin (POMC) and orexigenic neuropeptide Y (NPY)-containing ARC projections, it does so through distinct intracellular signaling pathways, and manipulation of these pathways produces divergent developmental outcomes for POMC and NPY neurons. The combined use of retrogradely transported tracers and histochemical visualization of neuronal terminals on PVH neurons shows that leptin preferentially increases the density of Agouti-related peptide (Agrp)containing inputs to preautonomic neurons in the $\mathrm{PVH}$; however, development of POMC inputs to these neurons is largely leptin independent (Fig. $3 B$ ). These anatomical findings suggest that the ability of leptin to specify patterns of neuronal connectivity in the hypothalamus during development may differentially impact distinct components of autonomic regulation. Leptin appears to exert these actions through direct action on ARC neurons that include promoting neurite extension, specifying patterns of innervation in hypothalamic targets, and influencing cell type-specific alterations in synaptic density.

\section{Conclusion}

These studies highlight the considerable recent progress that has been made in the analysis of the development of the thalamus and hypothalamus. The availability of new tools to rapidly manipulate gene function and characterize mutant phenotypes in these structures implies that this progress should accelerate in the years ahead. Perhaps the most exciting potential future application of these developmental studies will come from the physiological and behavioral analysis of animals in which the development or function of defined neural subpopulations has been disrupted. Although the anatomical structure of both the thalamus and hypothalamus is quite well characterized, the anatomical-physiological relationship between individual nuclei and neuronal subtypes and specific behaviors is still generally unclear. Both thalamic and hypothalamic anatomy is highly complex, and it is rarely possible to produce selective surgical lesions that only affect individual neuronal subtypes. Identifying the molecular codes that determine specification of neuronal subtypes in these structures may ultimately allow selective manipulation of the function of individual neural subtypes, and a detailed analysis of their contribution to perception and behavior.

Further analysis of thalamic and hypothalamic development is also likely to have considerable importance for human health. In addition to the well established effects of early nutritional status in controlling development of hypothalamic circuitry, it is also likely that genetic defects in the development of specific cell subtypes in thalamus and hypothalamus may directly lead to disorders of perception, metabolism, and homeostasis; this has already been reported for congenital obesity of both Mendelian and polygenic origin (Holder et al., 2000; Traurig et al., 2009). Coming years are likely to see the identification of numerous different cases where defects in development of diencephalic neurons are found to directly cause a range of human diseases.

\section{References}

Bénazet JD, Bischofberger M, Tiecke E, Gonçalves A, Martin JF, Zuniga A, Naef F, Zeller R (2009) A self-regulatory system of interlinked signaling feedback loops controls mouse limb patterning. Science 323:1050-1053. 
Bouret SG, Draper SJ, Simerly RB (2004) Trophic action of leptin on hypothalamic neurons that regulate feeding. Science 304:108-110.

Bulfone A, Puelles L, Porteus MH, Frohman MA, Martin GR, Rubenstein JL (1993) Spatially restricted expression of Dlx-1, Dlx-2 (Tes-1), Gbx-2, and Wnt-3 in the embryonic day 12.5 mouse forebrain defines potential transverse and longitudinal segmental boundaries. J Neurosci 13:3155-3172.

Dale JK, Vesque C, Lints TJ, Sampath TK, Furley A, Dodd J, Placzek M (1997) Cooperation of BMP7 and SHH in the induction of forebrain ventral midline cells by prechordal mesoderm. Cell 90:257-269.

Espinosa JS, Wheeler DG, Tsien RW, Luo L (2009) Uncoupling dendrite growth and patterning: single-cell knockout analysis of NMDA receptor 2B. Neuron 62:205-217.

Figdor MC, Stern CD (1993) Segmental organization of embryonic diencephalon. Nature 363:630-634.

Fragkouli A, Hearn C, Errington M, Cooke S, Grigoriou M, Bliss T, Stylianopoulou F, Pachnis V (2005) Loss of forebrain cholinergic neurons and impairment in spatial learning and memory in LHX7-deficient mice. Eur J Neurosci 21:2923-2938.

Holder JL Jr, Butte NF, Zinn AR (2000) Profound obesity associated with a balanced translocation that disrupts the SIM1 gene. Hum Mol Genet 9:101-108.

Iwasato T, Datwani A, Wolf AM, Nishiyama H, Taguchi Y, Tonegawa S, Knöpfel T, Erzurumlu RS, Itohara S (2000) Cortex-restricted disruption of NMDAR1 impairs neuronal patterns in the barrel cortex. Nature 406:726-731.

Kapsimali M, Caneparo L, Houart C, Wilson SW (2004) Inhibition of Wnt/ Axin/beta-catenin pathway activity promotes ventral CNS midline tissue to adopt hypothalamic rather than floorplate identity. Development 131:5923-5933.

Kataoka A, Shimogori T (2008) Fgf8 controls regional identity in the developing thalamus. Development 135:2873-2881.

Kiecker C, Lumsden A (2004) Hedgehog signaling from the ZLI regulates diencephalic regional identity. Nat Neurosci 7:1242-1249.

Kitamura K, Miura H, Yanazawa M, Miyashita T, Kato K (1997) Expression patterns of Brx1 (Rieg gene), Sonic hedgehog, Nkx2.2, Dlx1 and Arx during zona limitans intrathalamica and embryonic ventral lateral geniculate nuclear formation. Mech Dev 67:83-96.

Kurrasch DM, Cheung CC, Lee FY, Tran PV, Hata K, Ingraham HA (2007) The neonatal ventromedial hypothalamus transcriptome reveals novel markers with spatially distinct patterning. J Neurosci 27:13624-13634.

Lendvai B, Stern EA, Chen B, Svoboda K (2000) Experience-dependent plasticity of dendritic spines in the developing rat barrel cortex in vivo. Nature 404:876-881.

Macdonald R, Barth KA, Xu Q, Holder N, Mikkola I, Wilson SW (1995) Midline signalling is required for Pax gene regulation and patterning of the eyes. Development 121:3267-3278.

Manning L, Ohyama K, Saeger B, Hatano O, Wilson SA, Logan M, Placzek M (2006) Regional morphogenesis in the hypothalamus: a BMP-Tbx2 pathway coordinates fate and proliferation through Shh downregulation. Dev Cell 11:873-885.

Mori T, Yuxing Z, Takaki H, Takeuchi M, Iseki K, Hagino S, Kitanaka J,
Takemura M, Misawa H, Ikawa M, Okabe M, Wanaka A (2004) The LIM homeobox gene, L3/Lhx8, is necessary for proper development of basal forebrain cholinergic neurons. Eur J Neurosci 19:3129-3141.

Ohyama K, Das R, Placzek M (2008) Temporal progression of hypothalamic patterning by a dual action of BMP. Development 135:3325-3331.

Osorio J, Mazan S, Rétaux S (2005) Organisation of the lamprey (Lampetra fluviatilis) embryonic brain: insights from LIM-homeodomain, Pax and hedgehog genes. Dev Biol 288:100-112.

Puelles L, Rubenstein JL (1993) Expression patterns of homeobox and other putative regulatory genes in the embryonic mouse forebrain suggest a neuromeric organization. Trends Neurosci 16:472-479.

Puelles L, Rubenstein JL (2003) Forebrain gene expression domains and the evolving prosomeric model. Trends Neurosci 26:469-476.

Rebsam A, Seif I, Gaspar P (2002) Refinement of thalamocortical arbors and emergence of barrel domains in the primary somatosensory cortex: a study of normal and monoamine oxidase a knock-out mice. J Neurosci 22:8541-8552.

Ruiz i Altaba A (1998) Combinatorial Gli gene function in floor plate and neuronal inductions by Sonic hedgehog. Development 125:2203-2212.

Scholpp S, Wolf O, Brand M, Lumsden A (2006) Hedgehog signalling from the zona limitans intrathalamica orchestrates patterning of the zebrafish diencephalon. Development 133:855-864.

Scholpp S, Delogu A, Gilthorpe J, Peukert D, Schindler S, Lumsden A (2009) Her6 regulates the neurogenetic gradient and neuronal identity in the thalamus. Proc Natl Acad Sci U S A 106:19895-19900.

Shimogori T, Lee DA, Miranda-Angulo A, Yang Y, Wang H, Jiang L, Yoshida AC, Kataoka A, Mashiko H, Avetisyan M, Qi L, Qian J, Blackshaw S (2010) A genomic atlas of mouse hypothalamic development. Nat Neurosci 13:767-775.

Simerly RB (2008) Hypothalamic substrates of metabolic imprinting. Physiol Behav 94:79-89.

Szabó NE, Zhao T, Cankaya M, Theil T, Zhou X, Alvarez-Bolado G (2009) Role of neuroepithelial Sonic hedgehog in hypothalamic patterning. J Neurosci 29:6989-7002.

Tran PV, Lee MB, Marín O, Xu B, Jones KR, Reichardt LF, Rubenstein JR, Ingraham HA (2003) Requirement of the orphan nuclear receptor SF-1 in terminal differentiation of ventromedial hypothalamic neurons. Mol Cell Neurosci 22:441-453.

Traurig M, Mack J, Hanson RL, Ghoussaini M, Meyre D, Knowler WC, Kobes S, Froguel P, Bogardus C, Baier LJ (2009) Common variation in SIM1 is reproducibly associated with BMI in Pima Indians. Diabetes 58:1682-1689.

Vue TY, Bluske K, Alishahi A, Yang LL, Koyano-Nakagawa N, Novitch B, Nakagawa Y (2009) Sonic hedgehog signaling controls thalamic progenitor identity and nuclei specification in mice. J Neurosci 29:4484-4497.

Zhao Y, Guo YJ, Tomac AC, Taylor NR, Grinberg A, Lee EJ, Huang S, Westphal H (1999) Isolated cleft palate in mice with a targeted mutation of the LIM homeobox gene lhx8. Proc Natl Acad Sci U S A 96:15002-15006.

Zhao Y, Kwan KM, Mailloux CM, Lee WK, Grinberg A, Wurst W, Behringer RR, Westphal H (2007) LIM-homeodomain proteins Lhx1 and Lhx5, and their cofactor Ldb1, control Purkinje cell differentiation in the developing cerebellum. Proc Natl Acad Sci U S A 104:13182-13186. 